\title{
Primeiro registro de eversão vaginal em Psammobatis rutrum Jordan, 1891 (Rajiformes: Arhynchobatidae)
}

First record of vaginal eversion in Psammobatis rutrum Jordan, 1891 (Rajiformes: Arhynchobatidae)

Primer registro de eversión vaginal en Psammobatis rutrum Jordan, 1891 (Rajiformes: Arhynchobatidae)

Bruna Cabral Carneiro Batista

ORCID: https://orcid.org/0000-0002-2973-0248 Acervo Zoológico da Universidade Santa Cecília, Brasil E-mail: bruna.ccbatista@gmail.com

Helen Sadauskas Henrique ORCID: https://orcid.org/0000-0001-6988-3401 Laboratório de Biologia de Organismos Marinhos e Costeiros, Brasil E-mail: helen@unisanta.br

Matheus Marcos Rotundo

ORCID: https://orcid.org/0000-0003-1886-5320 Acervo Zoológico da Universidade Santa Cecília, Brasil E-mail:mmrotundo@unisanta.br

\begin{abstract}
Resumo
A externalização de órgãos internos em peixes tem sido registrada ao longo do tempo na literatura científica. Grande parte dos registros está relacionada à eversão ou protrusão do estômago e/ou intestino, devido principalmente ao barotrauma. Porém uma pequena parcela dos registros é referente a diversas causas naturais. O presente estudo teve como objetivo registrar a ocorrência de um raro evento de eversão vaginal na raia anã espinhosa Psammobatis rutrum. Em 15/06/2020 três exemplares (2 fêmeas e 1 macho) foram capturados como fauna acompanhante da pesca de parelha nas proximidades da Barra de Santos SP, com profundidade média de 27 metros. Dentre eles, uma exemplar fêmea apresentava uma protrusão via abertura cloacal. Através de necropsia, avaliação comparativa e estudo das vias reprodutivas foi identificado que o órgão externalizado era a parede vaginal. Embora existam muitos registros de externalização de órgãos de Elasmobranchii, principalmente relacionados ao comportamento natural de eversão do estômago e intestino, apenas um registro deste tipo de evento foi reportado para órgão reprodutivo. Não foi possível identificar a causa da eversão vaginal, porém tal evento está associado a um quadro de prolapso pélvico, que pode ter sido intensificado por causas naturais ou devido a captura.
\end{abstract}

Palavras-chave: Protrusão; Prolapso pélvico; Raia anã espinhosa.

\begin{abstract}
The externalization of internal organs in fish has been recorded over time in the scientific literature. Most of the records are related to the eversion or protrusion of the stomach and /or intestine, mainly due to barotrauma. However, a small portion of the records refer to several natural causes. The present study aimed to record the occurrence of a rare event of vaginal eversion in the spiny dwarf ray Psammobatis rutrum. On 06/15/2020 three specimens ( 2 females and 1 male) were captured as bycatch pair trawl in Barra de Santos SP, with an average depth of 27 meters. Among them, a female specimen presented a protrusion via the cloacal opening. Through necropsy, comparative evaluation and study of the reproductive pathways it was identified that the externalized organ was the vaginal wall. Although there are many records of externalization of Elasmobranchii organs, mainly related to the natural eversion behavior of the stomach and intestine, only one record of this type of event has been reported for the reproductive organ. It was not possible to identify the cause of vaginal eversion, but this event is associated with a pelvic prolapse, which may have been intensified by natural causes or due to capture.
\end{abstract}

Keywords: Protrusion; Pelvic prolapse; Spiny dwarf ray.

\section{Resumen}

La externalización de órganos internos en peces se ha registrado a lo largo del tiempo en la literatura científica. La mayoría de los registros están relacionados con la eversión o protrusión del estómago y/o intestino, principalmente por barotrauma. Sin embargo, una pequeña parte de los registros se refiere a varias causas naturales. El presente estudio tuvo como objetivo registrar la ocurrencia de un evento raro de eversión vaginal en la raya enana espinosa 
Psammobatis rutrum. El 15/06/2020 tres ejemplares (2 hembras y 1 macho) fueron capturados como fauna acompañante de pesca en pareja en las cercanías del Barra de Santos SP, con una profundidad promedio de 27 metros. Entre ellos, un ejemplar femenino presentó una protuberancia a través de la abertura cloacal. Mediante necropsia, evaluación comparativa y estudio de las vías reproductivas se identificó que el órgano exteriorizado era la pared vaginal. Aunque existen muchos registros de externalización de órganos de Elasmobranchii, principalmente relacionados con el comportamiento natural de eversión del estómago e intestino, solo se ha reportado un registro de este tipo de evento para el órgano reproductor. No fue posible identificar la causa de la eversión vaginal, pero este evento se asocia con un cuadro de prolapso pélvico, que puede haberse intensificado por causas naturales o por captura.

Palabras clave: Protrusión; Prolapso pélvico; Raya enana espinosa.

\section{Introdução}

A família Arhynchobatidae possui 13 gêneros e aproximadamente 104 espécies (Last, Stehmann, Séret \& Weigman, 2016). O gênero Psammobatis Günther, 1870 é composto por oito espécies endêmicas da América do Sul (Oceanos Pacífico e Atlântico), sendo quatro registradas no Brasil: P. bergi, P. extenta, P. lentiginosa e P. rutrum (Paragó, 2001; Gomes, Signori, Gadig \& Santos, 2010; Last et al., 2016; Fricke, Eschmeyer \& Van der Laan, 2021). A raia anã espinhosa, Psammobatis rutrum Jordan, 1891 distribui-se do Espírito Santo (Brasil) ao norte da Argentina, sob fundos de lama, areia, cascalho e rochoso, entre profundidades de 30 e 150 metros (Bernardes et al., 2005; Gomes et al., 2010; Last et al., 2016). Alcançam até $32 \mathrm{~cm}$ de comprimento e os machos atingem maturidade sexual entre 23 e $27 \mathrm{~cm}$. São ovíparas e depositam ovos pequenos (<30 mm) (Mabragaña et al., 2011; Last et al., 2016; Martins \& Oddone, 2017). Alimentam-se predominantemente de crustáceos e ocasionalmente de peixes e poliquetos (Viana, Valentin \& Vianna, 2017). É capturada como fauna acompanhante na pesca de arrasto e emalhe de fundo (Bernardes et al., 2005; Bornatowski, Abilhoa \& Charvet-Ameida, 2009; Schroeder, Pio, Bail, Lopes \& Wahrlich, 2014; Rocha \& Dias, 2015). Seu status de conservação global é caracterizado como menos preocupante (Pollom et al., 2020), enquanto no Brasil é classificado como dados deficientes (ICMBio, 2018).

A externalização (protrusão) de órgãos internos em peixes tem sido registrada ao longo do tempo na literatura científica (Andrews \& Bissell, 1934; Budker, 1971; Young, 1983; Moss, 1984; Crow \& Brock, 1993; Morales \& Dunker, 1999; Horodysky \& Graves, 2005; Schaller \& Dunker, 2005; Brunnschweiler, Nielsen \& Motta, 2011; Wiersma, Verhoog, Fowler \& Meekan, 2016; Weideli \& Papastamatiou, 2021). Tais registros podem ser classificados em dois tipos distintos: 1. Eversão: quando o órgão externalizado está revertido de seu plano natural e 2. Protrusão simples: quando o órgão é externalizado, total ou parcialmente, sem reversão. Estes eventos foram registrados tanto em ambientes cativos (Crow et al., 1990; Crow \& Brock, 1993; Morales \& Dunker, 1999; Henningsen, Whitaker \& Walker, 2005; Schaller \& Dunker, 2005; Tuttle et al., 2008; Christie, 2012; Schreiber \& Coco, 2017) como naturais (Brunnschweiler, Andrews, Southall, Pickering \& Sims 2005; Clark, Papastamatiou \& Meyer 2008; Wiersma et al., 2016; Weideli \& Papastamatiou, 2021).

Grande parte dos registros de externalização de órgãos de peixes está relacionada à eversão do estômago/ intestino e a protusão simples do intestino, devido ao estresse de captura e principalmente como consequência do barotrauma (Heithaus, 2001; Horodysky \& Graves, 2005; Markaida \& Sosa-Nishizaki, 2010; Brunnschweiler et al. 2011; Ellis, McCully-Phillips \& Poisson, 2016). A diferença de pressão, devido a alteração de profundidade durante a captura, causa a expansão da vesícula gasosa que promove o deslocamento dos órgãos internos, geralmente externalizando o estômago e/ou o intestino, além de outras consequências como exoftalmia, perda de escamas, hemorragia nos olhos e nadadeiras, etc (Rummer \& Bennett, 2005; StJohn \& Syers, 2005; Rummer, 2007; Jarvis \& Lowe, 2008; Stewart, 2008; Brown et al., 2010; Campbell, Tolan, Strauss \& Diamond, 2010; Brown et al., 2014; Pracheil, DeRolph, Schramm \& Bevelhimer, 2016; Veldhuizen, Berentsen, De Boer, Van De Vis \& Bokkers, 2018). Tais efeitos são amplamente relatados em pescarias de diferentes espécies, métodos e profundidades, incluindo a pesca amadora (esportiva) e profissional (redes de espera e de arrasto, e espinhéis) (Budker, 1971; 
Clark, 1985; Buckley \& Miller, 1994; Heithaus, 2001; Horodysky \& Graves, 2005; Markaida \& Sosa-Nishizaki, 2010; Brunnschweiler et al., 2011; Brown et al., 2016; Ellis et al., 2016; Veldhuizen et al., 2018).

Uma pequena parcela dos registros de externalização é referente a diversas causas naturais, como o comportamento de elasmobrânquios que evertem o estômago para liberação de itens não digeríveis (Budker, 1971; Andrews \& Young, 1993; Sims, Andrews \& Young, 2000; Brunnschweiler et al., 2005; Schreiber \& Coco, 2017), ou do intestino para a liberação de conteúdo constipado e/ou controle parasitário (Crow et al., 1990; Morales \& Dunker, 1999; Henningsen et al., 2005; Schaller \& Dunker, 2005; Clark et al., 2008; Christie, 2012; Wiersma et al., 2016; Schreiber \& Coco, 2017). Também podem estar associados a complicações de diferentes naturezas, como infeções (Gore, Hadfield \& Clayton, 2007; Tuttle et al., 2008), congestão (Tuttle et al., 2008) e torções intestinais (Morales \& Dunker, 1999). Independente da causa, mesmo quando o tempo de exposição é curto, estes eventos podem ocasionar a perda parcial do órgão por predação, lacerações e infecções devido ao contato com os dentes, ou ainda distúrbios respiratórios, gerando complicações severas a saúde e até mesmo a morte do indivíduo (Crow, Brock, Howe \& Linnon, 1991; Morales \& Dunker, 1999; Brunnschweiler et al., 2005; Henningsen et al., 2005; Tuttle et al., 2008; Christie, 2012; Weideli \& Papastamatiou, 2021).

Dentre os registros de externalização de órgãos em elasmobrânquios não relacionados ao estresse de captura e ao barotrauma, observa-se apenas um registro relacionado ao aparelho reprodutivo. Tal registro é referente a um exemplar de Pteroplatytrygon violacea (Myliobatiformes: Dasyatidae) no Aquário Nacional de Baltimore (EUA), onde os autores associaram à infecção fúngica (Gore et al., 2007). Assim, o presente estudo teve como objetivo registrar a ocorrência de um raro evento de eversão vaginal em peixes, especificamente na raia anã espinhosa Psammobatis rutrum (Rajiformes: Arhynchobatidae).

\section{Metodologia}

Em 15/06/2020 três exemplares (2 fêmeas e 1 macho) da raia anã espinhosa, Psammobatis rutrum (Fig. 1A) foram capturados como fauna acompanhante da pesca de parelha nas proximidades da Barra de Santos, em direção ao município de Praia Grande - SP (240' 40”S/ 46 23'46”O) com profundidade média de 27 metros. Posteriormente a captura, os exemplares foram acondicionados em gelo até o momento do desembarque (20/06/2020). A obtenção dos exemplares foi através do "Projeto Pró-pesca: pescando o conhecimento" que consiste na integração de pescadores e pesquisadores para avaliação de aspectos biológicos e pesqueiros das espécies capturadas pela atividade amadora (esportiva) e profissional (artesanal e industrial).

Em laboratório foi verificado que uma das fêmeas apresentava uma protrusão via abertura cloacal (Fig. 1B). Posteriormente a identificação realizada com base em Gomes et al. (2010) e Last et al. (2016), os exemplares foram mensurados no comprimento total e largura de disco ( $\mathrm{mm})$. A necropsia foi realizada apenas nas fêmeas e consistiu na análise sistematizada dos órgãos dos sistemas digestório e reprodutivo, além de observações sobre as características morfológicas externas. Os órgãos internos foram avaliados quanto ao posicionamento e proporção em relação ao corpo, sendo comparados entre os dois exemplares (com e sem protrusão) (Fig. 1 B-C). Após a avaliação dos órgãos internos, utilizando uma sonda nasogástrica curta $\left(n^{\circ} 04\right)$ foram identificadas as vias dos sistemas reprodutivo e gastrointestinal a partir da abertura cloacal e cavidade oro-branquial. Posteriormente a esta avaliação, os órgãos do sistema digestório foram cuidadosamente retirados, mantendo apenas aqueles relativos ao sistema reprodutivo, e assim uma análise comparativa foi realizada. 
Figura 1. Psammobatis rutrum Jordan, 1891 (Rajiformes: Arhynchobatidae). (A): visão dorsal, (B-C): visão ventral, sendo (B): exemplar com eversão vaginal $(E V)$ e $(C)$ : exemplar comparativo.

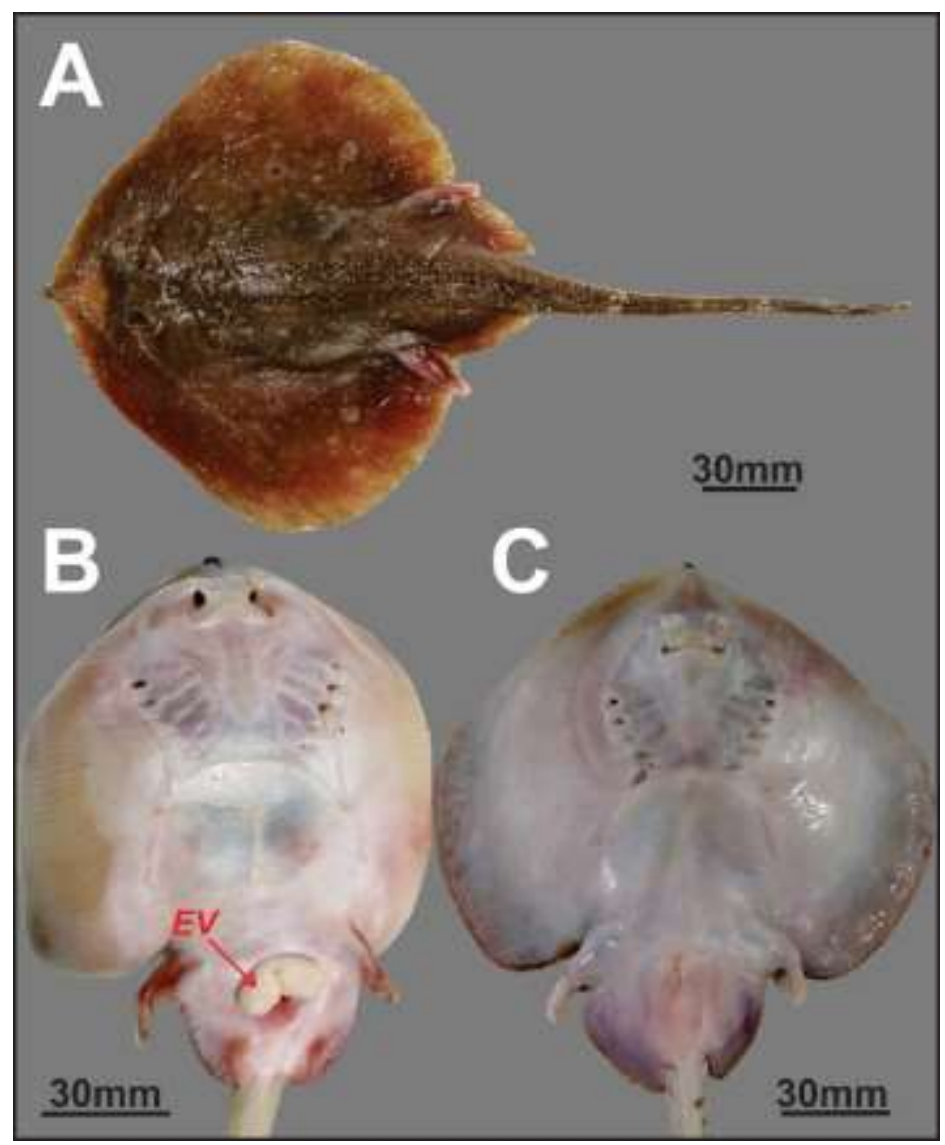

Fonte: Autores.

\section{Resultados}

O exemplar 1 (com protrusão, Fig. 1B, 2A-C) possuía comprimento total e largura de disco de $274 \mathrm{~mm} \mathrm{e} 139 \mathrm{~mm}$, enquanto a fêmea 2 (sem protrusão, Fig. 1C, 2 D-E e 3) $280 \mathrm{~mm}$ e $141 \mathrm{~mm}$ e o macho $250 \mathrm{~mm}$ e 115 mm, respectivamente. Os três exemplares apresentavam olhos translúcidos de tamanho proporcional (ausência de exoftalmia), coloração evidente (marrom-acastanhado), nadadeiras íntegras com disposição e proporcionalidade equivalentes, espinhos dorsais (tubérculos) completos, quando considerada a amplitude das linhas existentes e ausência de pontos hemorrágicos. A exemplar fêmea 1 e o macho apresentavam eritema principalmente nas cartilagens de sustentação das nadadeiras pélvicas (Fig. 1B, 2A-C), possivelmente correspondendo a queimaduras provenientes do processo de conservação. Os mesmos dois exemplares estavam com a musculatura tracionada, elevando as nadadeiras peitorais e comprimindo a região médio-dorsal (Fig. 1B, 2A-C). A contração muscular também ficou evidenciada externamente através da abertura das fendas branquiais, e internamente no exemplar fêmea1, pelo deslocamento parcial da coluna vertebral na região posterior da cavidade celomática, anteriormente a cérvix (Fig. 2C).

Quando comparadas (exemplares 1 e 2), verificamos que a disposição e proporcionalidade dos órgãos dos sistemas digestório (esôfago, estômago, intestino e fígado) e reprodutivo (ovários, ovidutos e úteros) estavam equivalentes. Os órgãos apresentavam mesma coloração, vascularização pouco evidente e ausência de áreas hemorrágicas (Fig. 3A-C).

Independente da via avaliada (cavidade oro-branquial e abertura cloacal), não foram observadas obstruções no sistema digestório. Tanto o estômago, quanto o intestino apresentavam conteúdo alimentar equivalentes a 50 e $45 \%$, respectivamente. 
No estômago foram identificados fragmentos de Polychaeta e Crustacea, enquanto no intestino não foi possível identificar os itens alimentares devido ao elevado grau de digestão.

O órgão externalizado apresentava coloração branca-amarelada, com leve enrijecimento ao toque e era composto por dois apêndices dispostos lateralmente em posição perpendicular ao eixo mediano central (Fig. 2A-C). Os dois apêndices estavam conectados na região basal, próximo da abertura cloacal e apresentavam três orifícios, um no apêndice direito e dois no esquerdo (Fig 2A-B). Os apêndices ocupavam apenas parte da abertura cloacal, na região anterior (Fig. 2A-B). Após uma incisão circundante à cavidade abdominal e posterior retirada dos órgãos do sistema gastrointestinal na exemplar fêmea 1, foi verificado que os apêndices externalizados correspondiam a parede vaginal, onde a cérvix encontrava-se parcialmente dobrada e evertida em sua porção anterior (Fig. 2C). Tal resultado ficou melhor evidenciado quando o mesmo procedimento foi realizado na exemplar fêmea 2 , onde também foi realizada uma incisão na cérvix, visualizando a parede vaginal e as duas aberturas para os úteros (direito e esquerdo) (Fig. 2D-E).

A avaliação das vias reprodutivas através dos orifícios localizados nos apêndices externalizados demostrou que um dos orifícios do apêndice esquerdo correspondia a uma dobra de tecido com fundo cego, enquanto os outros dois direcionavam a região uterina e ovários, comprovando que os apêndices correspondiam a parede vaginal (Fig. 2C). Embora a parede vaginal estivesse evertida, as vias não apresentavam obstruções até a região dos ovários.

Figura 2. Psammobatis rutrum (Rajiformes: Arhynchobatidae). (A-C): exemplar com eversão vaginal e (D-E): exemplar comparativo. $A v$ : abertura vaginal, $p u$ : poro urinário, $\mathrm{Cl}$ : abertura cloacal, $O v d$ : ovário direito, $O v e$ : ovário esquerdo, odt: oviduto, utd: útero direito, ute: útero esquerdo e $C V X$ : cérvix; barra: $20 \mathrm{~mm}$.

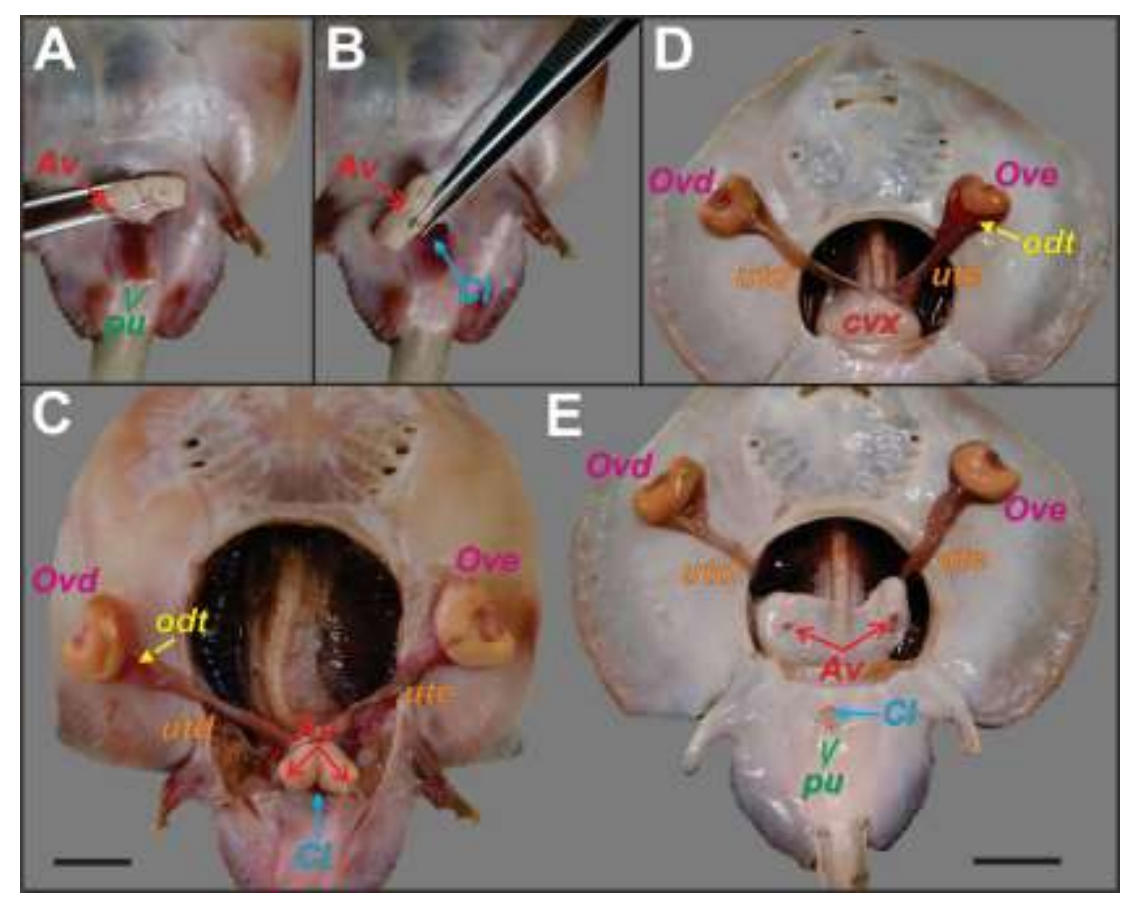

Fonte: Autores. 
Figura 3. Psammobatis rutrum (Rajiformes: Arhynchobatidae). (A): órgãos internos, (B): sistemas digestório e reprodutivo e (C): sistema reprodutivo. Fíg: fígado, est: estômago, int: intestino (válvula espiral), pu: poro urinário, Cl: abertura cloacal, Ovd: ovário direito, Ove: ovário esquerdo, utd: útero direito, ute: útero esquerdo e CVX: cérvix; barra: 30mm.

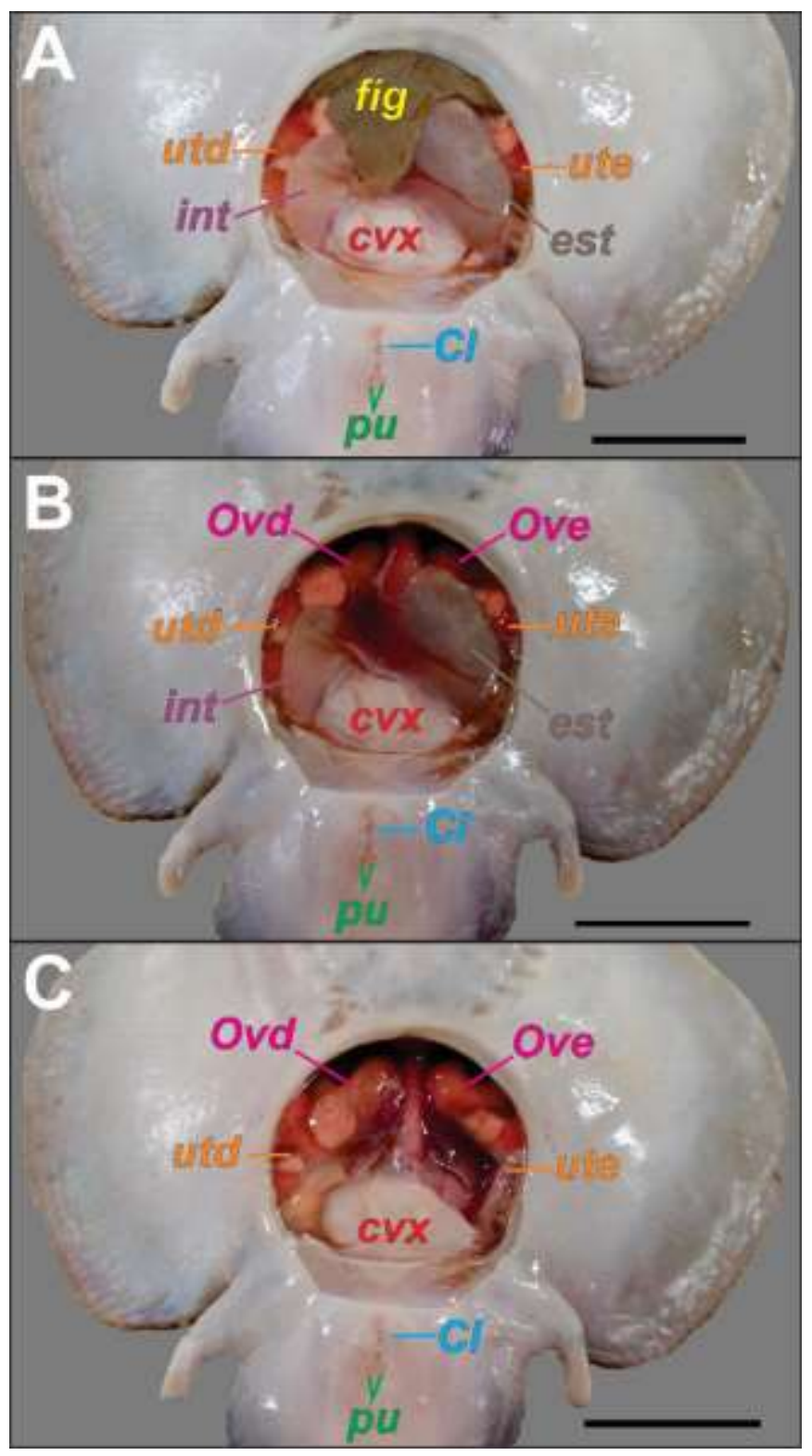

Fonte: Autores.

\section{Discussão}

A externalização de órgãos reprodutivos em peixes já foi reportada por Yanong, Curtis, Terrel \& Case (2003) em Antennarius striatus (Lophiiformes: Antennariidae). Os autores observaram uma massa ovígera (possivelmente as lamelas ovarianas e ovócitos) presa junto ao poro reprodutivo e associaram o evento ao diagnóstico histopatológico de micobacteriose sistêmica grave atípica, que causou a morte do exemplar. Outro registro foi reportado em Cyprinus carpio (Cyprinodontiformes: Cyprinidae) por Lewbart (2008). O autor evidenciou o tratamento clínico para a recuperação do exemplar, porém não relacionou a nenhuma causa evidente. A mesma relação de externalização com micobacteriose foi relatada em Xiphophorus montezumae (Cyprinodontiformes: Poeciliidae) por Arbuatti, Salda, Defourny \& Romanucci (2017). Através de análises histopatológicas, os autores relacionaram o prolapso ovariano à inflamação granulomatosa multifocal grave, caracterizada por micobacteriose. É importante salientar que as micobactérias estão presentes em vários corpos hídricos 
de diferentes salinidades e condições ambientais (Kirschner, Parker \& Falkinham III, 1992; Petrini, 2006; Regev et al., 2020), sendo a causa de inúmeras infecções em diversas espécies de peixes (Stoskopf, 1993; Smith, 1997; Francis-Floyd, 2011; Hashish et al., 2018; Regev et al., 2020).

Gore et al. (2007) observaram o prolapso de tecido reprodutivo em uma fêmea de Pteroplatytrygon violacea (Myliobatiformes: Dasyatidae) no Aquário Nacional de Baltimore (EUA) (Tab. 1). O exemplar foi submetido a tratamento com antibióticos e analgésicos, sendo monitorado os parâmetros hematológicos e bioquímicos. Através de ultrassonografia evidenciaram o acúmulo de fluído, espessamento e descamação da parede uterina. Com base na cultura do fluído uterino foi identificado a presença de hifas fúngicas que se propagaram até morte do exemplar. As análises histopatológicas deram suporte ao diagnóstico de endometrite micótica crônica severa difusa, sendo o fungo identificado como Paecilomyces lilacinus.

Embora o único registro observado sobre externalização de órgão reprodutivo em elasmobrânquios seja o estudo de Gore et al. (2007), técnicas veterinárias para casos clínicos são apresentadas em Sladky \& Clarke (2016), que também abordaram metodologias para eventos de protrusão gástrica e intestinal em animais cativos. Ainda sobre tratamentos veterinários, McDermott, Field, Mejia-Fava \& Clauss (2013) relataram a eversão gástrica e intestinal em elasmobrânquios como efeito colateral do tratamento de parasitoses com sulfonamidas, clindamicina, toltrazuril ou ponazuril (Tab. 1).

Tabela 1. Registros de externalização (protrusão) em elasmobrânquios (Ordem cronológica). Órgão (Est.: estômago e Int.: intestino), Ambiente (Cat.: cativeiro e Nat.: natural).

\begin{tabular}{|c|c|c|c|c|}
\hline Espécie & Família & Órgão & Ambiente & Referência \\
\hline Carcharhinus perezi & Carcharhinidae & Est. & Nat. & Budker (1971) \\
\hline$x$ & Carcharhinidae & Int. & Nat. & Compagno (1988) \\
\hline Carcharhinus galapagensis & Carcharhinidae & Int. & Cat. & Crow et al. (1990) \\
\hline Carcharhinus leucas & Carcharhinidae & Int. & Cat. & Crow et al. (1990) \\
\hline Carcharhinus melanopterus & Carcharhinidae & Int. & Cat. & Crow et al. (1990) \\
\hline Carcharhinus obscurus & Carcharhinidae & Int. & Cat. & Crow et al. (1990) \\
\hline Carcharhinus plumbeus & Carcharhinidae & Int. & Cat. & Crow et al. (1990) \\
\hline Negaprion acutidens & Carcharhinidae & Int. & Cat. & Crow et al. (1990) \\
\hline Negaprion brevirostris & Carcharhinidae & Int. & Cat. & Crow et al. (1990) \\
\hline Triaenodon obesus & Carcharhinidae & Int. & Cat. & Crow et al. (1990) \\
\hline Carcharhinus melanopterus & Carcharhinidae & Int. & Cat. & Crow et al. (1991) \\
\hline Carcharhinus melanopterus & Carcharhinidae & Int. & Cat. & Crow \& Brock (1993) \\
\hline Carcharhinus melanopterus & Carcharhinidae & Int. & Cat. & Morales \& Dunker (1999) \\
\hline Raja clavata & Rajidae & Est. & Cat. & Sims et al. (2000) \\
\hline Galeocerdo cuvier & Carcharhinidae & Est. & Nat. & Heithaus (2001) \\
\hline Carcharhinus perezi & Carcharhinidae & Est. & Nat. & Brunnschweiler et al. (2005) \\
\hline Pristis pectinata & Pristidae & Int. & Cat. & Henningsen et al. (2005) \\
\hline Carcharhinus melanopterus & Carcharhinidae & Int. & Cat. & Schaller \& Dunker (2005) \\
\hline Pteroplatytrygon violacea & Dasyatidae & Rep. & Cat. & Gore et al. (2007) \\
\hline Manta birostris & Mobulidae & Int. & Nat. & Clark et al. (2008) \\
\hline Carcharhinus taurus & Carcharhinidae & Est. & Cat. & $\begin{array}{c}\text { Tuttle et al. }(2008) \\
\text { Markaida \& Sosa-Nishizaki }\end{array}$ \\
\hline Prionace glauca & Carcharhinidae & Est. & Cat. & $(2010)$ \\
\hline Isurus oxyrinchus & Lamnidae & Est. & Nat. & Brunnschweiler et al.(2011) \\
\hline Hypanus americanus & Dasyatidae & Int. & Cat. & Christie (2012) \\
\hline Mustelis canis & Triakidae & Int. & Cat. & Christie (2012) \\
\hline
\end{tabular}




\begin{tabular}{lcccc}
\hline \multicolumn{1}{c}{ Espécie } & Família & Órgão & Ambiente & Referência \\
\hline Potamotrygon motoro & Potamotrygonidae & Int. & Cat. & Christie (2012) \\
Rhinobatos rhinobatos & Rhinobatidae & Int. & Cat. & Christie (2012) \\
Rhinoptera bonasus & Rhinopteridae & Int. & Cat. & Christie (2012) \\
Rhizoprionodon terraenovae & Carcharhinidae & Int. & Cat. & Christie (2012) \\
Sphyrna tiburo & Sphyrnidae & Int. & Cat. & Christie (2012) \\
Squalus acanthias & Squalidae & Int. & Cat. & Christie (2012) \\
Rhinoptera bonasus & Rhinopteridae & Int. & Cat. & McDermott et al. (2013) \\
Notorynchus cepedianus & Hexanchidae & Int. & Nat. & Wiersma et al. (2016) \\
Rhincodon typus & Rhincodontidae & Est./Int. & Cat. & Schreiber \& Coco (2017) \\
Rhina ancylostomus & Rhinidae & Int. & Cat. & Dvornak, Hanna, Hazeres, \& \\
& & & & Brehob (2017) \\
Carcharhinus longimanus & Carcharhinidae & Int. & Nat. & Weideli \& Papastamatiou (2021) \\
Psammobatis rutrum & Arhynchobatidae & Rep. & Nat. & presente estudo \\
\hline
\end{tabular}

Fonte: Autores.

No presente registro, as causas da eversão da parede vaginal não ficaram muito claras. Não foi possível realizar análises de cultura ou histopatológicas devido ao extenso período entre a captura e a análise do exemplar, o que poderia interferir nos resultados. Mesmo assim, consideramos importante realizar algumas hipóteses sobre o evento.

A protrusão como consequência de barotrauma em elasmobrânquios já foi relatada anteriormente. Mesmo sem possuir vesícula gasosa, uma pressão de $200 \mathrm{~mm} \mathrm{Hg}$ exercida pela expansão gasosa na cavidade peritoneal de elasmobrânquios pode resultar na eversão gástrica (Sims et al., 2000). Embora não seja possível descartar totalmente o efeito do barotrauma como causador da eversão vaginal observada, consideramos que a presença de outros exemplares da mesma espécie em condições normais, juntamente com a ausência de outras complicações características ao barotrauma (como exoftalmia e hemorragia nos olhos e nadadeiras) sejam indícios adequados para desconsiderar o efeito da pressão como causa da eversão observada.

O enrijecimento (tracionamento) da musculatura da exemplar fêmea 1 e do macho correspondem ao processo de rigidez cadavérica (rigor mortis) devido ao esgotamento de trifosfato de adenosina (ATP) (Soares \& Gonçalves, 2012). Após a morte, os compostos orgânicos tendem a se hidrolisar, sendo o glicogênio o mais rápido, causando o acúmulo de ácido láctico nos músculos e a redução do pH (Beirão et al., 2004; Siqueira, Godoy, Moraes \& Prata, 2018). Este processo está relacionado a complexas reações bioquímicas que envolvem desde da morte por asfixia (ausência de $\mathrm{O}_{2}$ ), até uma cadeia consequente relacionada ao trifosfato de adenosina (ATP), adenosina difosfato (ADP), glicogênio, miosina e ácido láctico (Oetterer, Galvão, \& Savay-Da-Silva, 2014). Tal enrijecimento é variável entre espécies e sofre alterações devido à temperatura, manipulação, tamanho e condições físicas do organismo capturado (Zanini, Martins, Torres \& Tobias, 2001; Moura et al., 2018; Silva-Presenza et al., 2020).

Outra possível causa está relacionada a anomalias congênitas do sistema reprodutivo. Diferentes anomalias já foram reportadas em elasmobrânquios, incluindo alterações cromáticas (Ex.: Sandoval-Castillo, Mariano-Melendez \& VillavicencioGarayzar, 2006; Bottaro, Ferrando, Gallus, Girosi \& Vacchi, 2008; Jones, Hoffmayer \& Gropp, 2016; Quigley, Carlos, BarrosGarcia \& MacGbahann, 2018; Lara-Mendoza \& Guerra-Jiménez, 2020), morfológicas (Ex.: El Kamel-Moutalibi, Mnasri, Boumaïza, Reynaud \& Capapé, 2011; Pastén-Marambio, Hevia-Hormazábal, Acuña \& Vega, 2018; Marouani, Jarray, Karaa \& Jarboui, 2019; Ranjith, et al., 2019; Mulas et al., 2020; Rolim, Rotundo, Stabile \& Vaske-Júnior, 2020) e reprodutivas (Oddone \& Amorim, 2008; Ribeiro-Prado, Oddone, Amorim \& Capapé, 2009; Capapé, El Kamel-Moutalibi, Mnasri, Boumaïza \& Reynaud, 2012; Costa, Borges \& Capapé, 2013; Hendon, Koester, Hoffmayer, Driggers III \& Cicia, 2013; Santander-Neto \& Lessa, 2013; Matta, 2015; Moore, 2015; Straube, Lampert, Geiger, Weiss \& Kirchhauser, 2016; Rafrafi- 
Nouira, El Kamel-Moutalibi, Amor, Amor, \& Capapé, 2017; Zhao, Chen, Jiang, Yang, \& Xiao, 2017; Ehemann \& GonzálezGonzález, 2018; Burgos, Gonzalez, Falla, \& Escalon, 2019; Capapé, Kassar, Reynaud, \& Hemida, 2019; Sen, Dash, Valappil, Kizhakudan, \& Chakraborty, 2020; Silva \& Silva-Casas, 2020). Grande parte das anomalias relacionadas aos órgãos reprodutivos está relacionada a intersexualização, deformidades ou ausência de cláspers. Embora as características de anomalias sejam bem específicas em cada indivíduo, não observamos nenhuma similaridade com os registros disponíveis (supracitados). Não foi observado qualquer indício de anomalia nos órgãos do exemplar com eversão vaginal, mesmo em relação ao sistema reprodutivo. Tal afirmação pode ser suportada pela análise comparativa com o exemplar de porte similar, mesmo sexo, estágio de desenvolvimento ovariano, localidade e artefato de captura. Logicamente não é possível afirmar que não se trata de uma anomalia congênita, porém achamos pouco provável, ainda que o espessamento da parede vaginal externalizada seja diferenciado.

Uma terceira hipótese para a eversão da parede vaginal está relacionada ao quadro de prolapso pélvico, que consiste na perda do suporte vaginal realizado por tecido conjuntivo junto a musculatura pélvica (Schimpf \& Tulikangas, 2005; Jelovsek, Maher \& Barber, 2007).

Embora muitas causas sejam atribuídas aos eventos de prolapso pélvico, um quadro associativo está relacionado a formação de biofilme bacteriano (e infecções), que consiste em uma comunidade de microrganismos aderentes dentro de uma matriz composta por substâncias poliméricas extracelulares que se formam em diferentes superfícies internas corporais (Gould et al., 2021). Tal relação reafirma o observado por Gore et al. (2007) para Pteroplatytrygon violacea (Myliobatiformes: Dasyatidae).

Outro fator responsável por este quadro pode estar associado a dificuldades durante o parto/ ovoposição (distocia), como observado para diferentes grupos zoológicos, incluindo peixes (Wildgoose, 2000; Asiimwe et al., 2014; Gal, Baska, Farkas, Mándoki, \& Szabó, 2014; Manjunatha, Girish \& Narayanaswamy, 2016; Staggs, 2016; Van Zeeland, 2016; Chaudhari et al., 2018; Leite, Wosnick, Giareta \& Freire, 2020). Assim, no presente estudo também podemos considerar que a eversão da parede vaginal pode ter sido resultante de um processo de prolapso pélvico intensificado por um evento de cópula malsucedido, postura repentina de ovos devido ao estresse, ou ainda à distocia, geralmente relacionada ao tamanho e posicionamento anormal de ovos/ embriões ou a problemas de contração abdominais (Noakes, Parkinson, England \& Arthur, 2001; Henningsen, Smale, Garner \& Kinnunen, 2004; Eid-Arimoku \& Trompetas, 2011; George et al., 2017; Wosnick, Adams \& Freire 2018; Wosnick et al., 2019; Akpan, Akpanika, Nwagbara, Asibong, \& Etuk 2019; Leite et al., 2020).

\section{Considerações Finais}

Embora não tenha sido possível determinar a causa da eversão da parede vaginal do exemplar analisado de raia anã espinhosa, Psammobatis rutrum (Rajiformes: Arhynchobatidae), considera-se que tal evento esteja relacionado a um quadro de prolapso pélvico que pode ter sido agravado por diferentes causas naturais e/ou em decorrência de barotrauma devido à captura. Assim, o presente registro, evidencia a necessidade de estudos que objetivem a compreensão de tais eventos junto a frota pesqueira amadora e profissional, ou mesmo em exemplares cativos.

\section{Agradecimentos}

Os autores agradecem a todos os pescadores do "Projeto Pro-pesca: pescando o conhecimento" pela doação dos exemplares. Também agradecem ao Prof. Dr. Otto Bismarck Fazzano Gadig (Universidade Estadual Paulista - UNESP) pela confirmação taxonômica e preciosas sugestões. 


\section{Referências}

Akpan, U.; Akpanika, C.; Nwagbara, V.; Asibong, U. \& Etuk, S. (2019). Uterine Rupture with Evisceration of Intestines through the Vagina during Labour. Case reports in obstetrics and gynecology, 1-3. https://doi.org/10.1155/2019/5234641

Andrews, E. \& Bissell, A. (1934). Comparative studies of hernia in man and animals. The Journal of Urology, 31(6), 839-866. https://doi.org/10.1016/S0022$5347(17) 72442-1$

Andrews, P. L. R. \& Young, J. Z. (1993). Gastric motility patterns for digestion and vomiting evoked by sympathetic nerve stimulation and 5hydroxytryptamine in the dogfish Scyliorhinus canicula. Philosophical Transactions of the Royal Society B, 342, 363-380. https://doi.org/10.1098/rstb.1993.0165

Arbuatti, A; Salda, L. D.; Defourny, S. V. P. \& Romanucci, M. (2017). Ovarian prolapse associated with severe granulomatous hepatitis in an adult female Montezuma swordtail, Xiphophorus montezumae (Jordan \& Snyder, 1899). Scientific Poster, III SIVAE International Meeting, Rimini, 26-28. http://hdl.handle.net/11575/99980

Asiimwe, C.; Reynolds, V.; Zziwa, P.; Mugabe, T.; Walumbe, W.; Muhanguzi, G.; Babweteera, F. \& Zuberbühler, K. (2014). Vaginal Prolapse in a Handicapped, Multiparous Wild Chimpanzee in Budongo Forest Reserve, Uganda. African Primates, 9, 45-50. http://www.primate-sg.org/storage/africanprimates-journal/volume-9/African\%20Primates\%209\%202014\%20LR.pdf

Beirão, L. H.; Teixeira, E.; Batista, C. R. V.; Santo, M. L. E.; Damian, C. \& Meinert, E. M. (2004). Tecnologia pós-captura de pescado e derivados. In: Poli, C.R.; Polli, A.T.B.; Andreatta, E.R. \& Beltrame, E. Aqüicultura: Experiências Brasileiras. Florianópolis: Multitarefa, p.407-442.

Bernardes, R. Á.; Figueiredo, J. L.; Rodrigues, A. R.; Fischer, L. G.; Vooren, C. M.; Haimovici, M. \& Rossi-Wongtschowski, C. L. D. B. (2005). Peixes de zona econômica exclusiva da região sudeste-sul do Brasil: Levantamento com armadilhas, pargueiras e rede de arrasto de fundo. Universidade de São Paulo, São Paulo. 295p.

Bornatowski, H.; Abilhoa, V. \& Charvet-Ameida, P. (2009). Elasmobranchs of the Paraná coast, southern Brazil, south-western Atlantic. Marine Biodiversity Records, 2, 1-6. https://doi.org/10.1017/S1755267209990868

Bottaro, M.; Ferrando, S.; Gallus, L.; Girosi, L. \& Vacchi, M. (2008). First record of albinism in the deep-water shark Dalatias licha. Marine Biodiversity Records, 1, 1-4. https://doi.org/10.1017/S1755267205001156

Brown, I.; Sumpton, W.; McLennan, M.; Mayer, D.; Campbell, M.; Kirkwood, J.; Butcher, A.; Halliday, I.; Mapleston, A.; Welch, D.; Begg, G. A. \& Sawynok, B. (2010). An improved technique for estimating short-term survival of released line-caught fish, and an application comparing barotrauma-relief methods in red emperor (Lutjanus sebae Cuvier, 1816). Journal of Experimental Marine Biology and Ecology, 385(1-2), 1-7. https://doi.org/10.1016/j.jembe.2010.01.007

Brown, R. S.; Colotelo, A. H.; Pflugrath, B. D.; Boys, C. A.; Baumgartner, L. J.; Deng, Z. D.; Silva, L. G. M.; Brauner, C. J.; Mallen-Cooper, M.; Phonekhampeng, O.; Thorncraft, G. \& Singhanouvong, D. (2014). Understanding barotrauma in fish passing hydro structures: a global strategy for sustainable development of water resources. Fisheries, 39, 108-122. https://doi.org/10.1080/03632415.2014.883570

Brunnschweiler, J. M.; Andrews, P. L. R.; Southall, E. J.; Pickering, M. \& Sims, D. W. (2005). Rapid voluntary stomach eversion in a free-living shark. Journal of the Marine Biological Association of the United Kingdom, 85, 1141-1144. https://doi.org/10.1017/S0025315405012208

Brunnschweiler, J. M.; Nielsen, F. \& Motta, P. (2011). In situ observation of stomach eversion in a line-caught Shortfin Mako (Isurus oxyrinchus). Fisheries Research, 109(1), 212-216. https://doi.org/10.1016/j.fishres.2011.02.005

Buckley, T. W. \& Miller, B. S. (1994). Feeding habits of yellowfin tuna associated with fish aggregation devices in American Samoa. Bulletin of Marine Science, 55(2-3), 445-459. https://www.ingentaconnect.com/contentone/umrsmas/bullmar/1994/00000055/f0020002/art00017\#

Budker, P. (1971). The life of sharks. Columbia University Press, New York, EUA. 222p.

Burgos, M. I.; Gonzalez, L. D. V. G.; Falla, P. A. M. \& Escalon, V. H. C. (2019). First record of monoclasper in the Banded guitarfish, Zapteryx exasperata in the Gulf of California, Mexico. CICIMAR Oceánides, 34(1), 41-44. http://dx.doi.org/10.37543/oceanides.v34i1.236

Campbell, M. D.; Tolan, J.; Strauss, R. \& Diamond, S. L. (2010). Relating angling-dependent fish impairment to immediate release mortality of red snapper (Lutjanus campechanus). Fisheries Research, 106(1), 64-70. https://doi.org/10.1016/j.fishres.2010.07.004

Capapé, C.; El Kamel-Moutalibi, O.; Mnasri, N.; Boumaïza, M. \& Reynaud, C. (2012). A case of hermaphroditism in Tortonese's stingray, Dasyatis tortonesei (Elasmobranchii: Rajiformes: Dasyatidae) from the Lagoon of Bizerte, Tunisia. Acta Ichthyologica et Piscatoria, 42(2), 141. http://dx.doi.org/10.3750/AIP2011.42.2.08

Capapé, C.; Kassar, A.; Reynaud, C. \& Hemida, F. (2019). Atypical characteristics in blackmouth catshark, Galeus melastomus (Chondrichthyes: Scyliorhinidae) from the Algerian coast (southern Mediterranean Sea). Thalassia salentina, 41, 23-32. http://dx.doi.org/10.1285/i15910725v41p23

Chaudhari, N. F.; Modi, L. C.; Patel, C. M.; Raval, A. P.; Kataria, M. A. \& Sorathiya, L. M. (2018). A Case of Post Partum Cervicovaginal Prolapse in Surti Buffaloes. Veterinary Research International, 6(04), 80-81.https://www.researchgate.net/publication/335826277_A_Case_of_Post_Partum_Cervicovaginal_ Prolapse_in_Surti_Buffaloes

Christie, B. L. (2012). Intestinal eversion in the Atlantic sharpnose shark, Rhizoprionodon terraenovae, a behavior ubiquitous among elasmobranch fishes? Gulf of Mexico Science, 1-2, 50-53. http://dx.doi.org/10.18785/goms.3001.08

Clark, M. R. (1985). The food and feeding of seven fish species from the Campbell Plateau, New Zealand. New Zealand Journal of Marine and Freshwater Research, 19(3), 339-363. https://doi.org/10.1080/00288330.1985.9516100 
Clark, T. B.; Papastamatiou, Y. P. \& Meyer, C. G. (2008). Intestinal eversion in a free-ranging manta ray (Manta birostris). Coral Reefs, $27,61$. http://dx.doi.org/10.1007/s00338-007-0305-z

\section{Compagno, L. J. V. (1988). Sharks of the Order Carcharhiniformes. The Blackburn Press, Caldwell, New Jersey, EUA.}

Costa, M. E.; Borges, T. C. \& Capapé, C. (2013). Cases of abnormal hermaphroditism in velvet belly Etmopterus spinax (Chondrichthyes Etmopteridae) from the southern coast of Portugal. Cahiers de Biologie Marine, 54, 309-317. https://dx.doi.org/10.21411/CBM.A.F84B46D1

Crow, G. L. \& Brock. J. A. (1993). The use of gentamicin sulfate therapy in a captive blacktip reef shark (Carcharhinus melanopterus) with intestinal biting syndrome. Zoo Biology, 12, 479-482. https://doi.org/10.1002/zoo.1430120509

Crow, G. L.; Brock, J. A.; Howe, J. C. \& Linnon, B.E. (1991). Shark bite wounds of the valvular intestine: the cause of an acute mortality syndrome of captive blacktip reef sharks, Carcharhinus melanopterus. Zoo Biology, 10, 457-463. https://doi.org/10.1002/zoo.1430100604

Crow, G. L.; Howe, J. C.; Uchida, S.; Kamolnick, S.; Wisner, M. G. \& Caira, J. N. (1990). Protrusion of the valvular intestine through the cloaca in sharks of the family Carcharhinidae. Copeia, 1, 226-229. https://doi.org/10.2307/1445839

Dvornak, M. J.; Hanna, J.; Hazeres, J. \& Brehob, S. (2017). Husbandry of Bowmouth Guitarfish, Rhina ancylostoma In: Smith, M.; Warmolts, D.; Thoney, D.; Hueter, R.; Murray, M. \& Ezcurra, J. (2017). The elasmobranch husbandry manual II: recent advances in the care of sharks, rays and their relatives. The Ohio Biological Survey, Columbus. p.67-74.

Ehemann, N. R. \& González-González, L. (2018). First record of a single-clasper specimen of Pseudobatos percellens (Elasmobranchii: Rhinopristiformes: Rhinobatidae) from the Caribbean Sea, Venezuela. Acta Ichthyologica et Piscatoria, 48(3), 235-240. http://dx.doi.org/10.3750/AIEP/02341

Eid-Arimoku, L. \& Trompetas, V. (2011). Postcoital vaginal rupture after hysterectomy presenting as generalised peritonitis. The Annals of The Royal College of Surgeons of England, 93(7), e136-e137. https://dx.doi.org/10.1308\%2F147870811X602140

El Kamel-Moutalibi, O.; Mnasri, N.; Boumaïza, M.; Reynaud, C. \& Capapé, C. (2011). Abnormalities in common torpedos, Torpedo torpedo (Chondrichthyes: Torpedinidae) from the lagoon of Bizerte (northern Tunisia, Central Mediterranean). Annales Series Historia Naturalis, 20(2), 181-190. https://zdjp.si/wp-content/uploads/2015/12/ASHN-21-2011-2_El-Kamel.pdf

Ellis, J.R.; McCully-Phillips, S.R. \& Poisson, F. (2016). A review of capture and post release mortality of elasmobranchs. Journal of Fish Biology, 90(3), 653722. https://doi.org/10.1111/jfb.13197

Francis-Floyd, R. (2011). Mycobacterial infections of fish. Southern Regional Aquaculture Center. United States Departamento of Agriculture. National Institute of Food and Agriculture. 4706, 12p.

Fricke, R.; Eschmeyer, W. N. \& Van der Laan, R. (2021). Eschmeyer's Catalog of Fishes: Genera, Species, References http://researcharchive.calacademy.org/research/ichthyology/catalog/fishcatmain.asp

Gal, J.; Baska, F.; Farkas, S. L.; Mándoki, M. \& Szabó, E. (2014). Egg retention and subsequent autointoxication in a shell-dwelling Lamprologus ocellatus (Steindachner, 1909) stock. Magyar Állatorvosok Lapja, 136(10), 615-617. https://www.cabdirect.org/cabdirect/abstract/20143371051

George, R. H., Haywood, P., Steeil, J., Aczm, D., Box, P. O., Baine, K., \& Avian, D. A. (2017). Diagnosis and treatment of common reproductive problems in elasmobranchs. The elasmobranch husbandry manual II: Recent advances in the care of sharks, rays and their relatives, The Ohio Biological Survey, Columbus 357-362.

Gomes, U.L.; Signori, C.N.; Gadig, O.B.F. \& Santos, H.R.S. (2010). Guia para identificação de tubarões e raias do Rio de Janeiro. Technical Books, Rio de Janeiro. 234p.

Gore, S. R.; Hadfield, C. A. \& Clayton, L. A. (2007). Fungal endometritis in a pelagic stingray (Dasyatis violacea). International association for aquatic animal medicine. http://apputil/content/defaultadv1.aspx?pId=11235\&meta=generic\&id=3980776.

Gould, F. G.; Carey, M. P.; Plummer, E. L.; Murray, G. L.; Danielewski, J. A.; Tabrizi, S. N. \& Garland, S. M. (2021). Bacterial biofilm formation on vaginal ring pessaries used for pelvic organ prolapse. International Urogynecology Journal, 1-9. https://doi.org/10.1007/s00192-021-04717-x

Hashish, E.; Merwad, A.; Elgaml, S.; Amer, A.; Kamal, H.; Elsadek, A.; Marei, A. \& Sitohy, M. (2018). Mycobacterium marinum infection in fish and man: epidemiology, pathophysiology and management: a review. The Veterinary Quarterly, 38(1), 35-46. https://doi.org/10.1080/01652176.2018.1447171

Heithaus, M. R. (2001). The biology of tiger sharks, Galeocerdo cuvier, in Shark Bay, Western Australia: sex ratio, size distribution, diet, and seasonal changes in catch rates. Environmental Biology of Fishes, 61, 25-36. https://doi.org/10.1023/A:1011021210685

Hendon, J. M.; Koester, D. M.; Hoffmayer, E. R.; Driggers III, W. B. \& Cicia, A. M. (2013). Occurrence of an intersexual blacktip shark in the northern Gulf of Mexico, with notes on the standardization of classifications for this condition in elasmobranchs. Marine and Coastal Fisheries, 5(1), 174-180. https://doi.org/10.1080/19425120.2013.799618

Henningsen, A. D.; Smale, M.; Garner, R. \& Kinnunen, N. (2004). Reproduction, embryonic development, and reproductive physiology of elasmobranchs. In: Smith, M.; Warmolts, D.; Thoney, D. \& Hueter, R. The elasmobranch husbandry manual: captive care of sharks, rays and their relatives. Columbus: Ohio Biological Survey. 585p.

Henningsen, A. D.; Whitaker, B. R. \& Walker, I. D. (2005). Protrusion of the valvular intestine in captive smalltooth sawfish and comments on pristid gastrointestinal anatomy and intestinal valve types. Jornal of Aquatic Animal Health, 17, 289-295. https://doi.org/10.1577/H04-063.1

Horodysky, A. Z. \& Graves, J. E. (2005). Application of pop-up satellite archival tag technology to estimate postrelease survival of white marlin (Tetrapturus albidus) caught on circle and straight-shank ("J") hooks in the western North Atlantic recreational fishery. Fishery Bulletin, 103, 84-96. http://aquaticcommons.org/id/eprint/9643 
ICMBio - Instituto Chico Mendes de Conservação da Biodiversidade (2018). Livro Vermelho da Fauna Brasileira Ameaçada de Extinção: Volume I, ICMBio/MMA. Brasília. 492p.

Jarvis, E. T. \& Lowe, C. G. (2008). The effects of barotrauma on the catch-and-release survival of southern California nearshore and shelf rockfish (Scorpaenidae, Sebastes spp.). Canadian Journal of Fisheries and Aquatic Sciences, 65, 1286-1296. http://dx.doi.org/10.1139/F08-071

Jelovsek, J. E.; Maher, C. \& Barber, M.D. (2007). Pelvic organ prolapse. The Lancet, 369(9566), 1027-1038. https://doi.org/10.1016/S0140-6736(07)60462-0

Jones, C. M.; Hoffmayer, E. R. \& Gropp, R. P. (2016). First record of a leucistic Narcine bancrofti (Elasmobranchii, Narcinidae) from the northern Gulf of Mexico. Cybium, 40(3), 249-251. https://doi.org/10.26028/cybium/2016-403-008

Kirschner, R. A.; Parker, B. C. \& Falkinham III, J.O. (1992). Epidemiology of infection by nontuberculous mycobacteria: Mycobacterium avium, Mycobacterium intracellulare, and Mycobacterium scrofulaceum in acid brown-water swamps of the southeastern United States and their association with environmental variables. American Review of Respiratory Disease, 145, 271-275. https://doi.org/10.1164/ajrccm/145.2_pt_1.271

Lara-Mendoza, R. E. \& Guerra-Jiménez, L. A. (2020). Record of albinism in the Smooth Butterfly ray Gymnura micrura (Rajiformes, Gymnuridae) from the southeastern Gulf of Mexico. Pan-American Journal of Aquatic Sciences, 15(1), 33-38. https://panamjas.org/pdf_artigos/PANAMJAS_15(1)_33-38.pdf

Last, P. R.; Stehmann, M. F. W.; Séret, S. \& Weigmann (2016). Softnose Skates. Family Arhynchobatidae. In: Last, P. R.; White, W. T.; Carvalho, M. R.; Séret, B.; Stehmann, M. F. W. \& Naylor, G. J. P. Rays of the World. Comstock Publishing, p.734-955.

Leite, R. D.; Wosnick, N.; Giareta, E. P. \& Freire, C. A. (2020). Evidence of dystocia in a free-ranging Cownose Ray Rhinoptera bonasus (Elasmobranchii, Rhinopteridae). Boletim do Laboratório de Hidrobiologia, 30(2), 1-6. http://www.periodicoseletronicos.ufma.br/index.php/blabohidro/article/view/14147/ 7776

Lewbart, G. A. (2008). Ornamental Fish. In: Rosenthal, K.; Forbes, N.; Frye, F. \& Lewbart, G. A. Rapid Review of Exotic Animal Medicine and Husbandry: Pet Mammals, Birds, Reptiles, Amphibians and Fish. Manson Publishing/ The Veterinary Press. CRC, p.244.

Mabragaña, E.; Figueroa, D. E.; Scenna, L. B.; Díaz de Astarloa, J. M.; Colonello, J. H. \& Delpiani, G. (2011). Chondrichthyan egg cases from the south-west Atlantic Ocean. Journal of Fish Biology, 79, 1261-1290. https://doi.org/10.1111/j.1095- 8649.2011.03111.x

Manjunatha, K.; Girish, B. C. \& Narayanaswamy, H. D. (2016). Dystocia in a ewe due to dicephalic fetus and its successful alleviation. North-East Veterinarian, 16(3), 8-10. https://www.cabdirect.org/cabdirect/abstract/20183001211

Markaida, U. \& Sosa-Nishizaki, O. (2010). Food and feeding habits of the blue shark Prionace glauca caught off Ensenada, Baja California, Mexico, with a review on its feeding. Jornal of the Marine Biological Association of the United Kingdom, 90, 977-994. https://doi.org/10.1017/S0025315409991597

Marouani, S.; Jarray, F. B.; Karaa, S. \& Jarboui, O. (2019). Morphological Abnormalities in Rajidae Species (Chondrichthyes) From the Gulf of Gabès (Tunisia, Central Mediterranean Sea): What Causes? Advances in Oceanography \& Marine Biology, 1(2), 1-5. http://dx.doi.org/10.33552/AOMB.2019.01.000507

Martins, M. F. \& Oddone, M.C. (2017). Reproductive biology of Psammobatis rutrum (Chondrichthyes: Arhynchobatidae) in south Brazil, south-west Atlantic. Journal of Fish Biology, 91(2), 443-459. http://dx.doi.org/10.1111/jfb.13350

Matta, M. E. (2015). Reproductive biology of the Alaska skate Bathyraja parmifera, with comments on an intersexual individual. Journal of Fish Biology, 87(3), 664-678. https://doi.org/10.1111/jfb.12747

McDermott, A.; Field, C.; Mejia-Fava, J. \& Clauss, T. (2013). Medical and surgical management of cloacal prolapses in cownose rays (Rhinoptera bonasus) undergoing treatment for Eimeria southwelli infection. Proceedings of the International Association for Aquatic Animal Medicine Conference, San Francisco, California, USA. p.276-277. https://alpha.vin.com/apputil/content/defaultadv1.aspx?pId=11375\&id=5768614

Moore, A. B. M. (2015). Morphological abnormalities in elasmobranchs. Journal of Fish Biology, 87(2), 465-471. https://doi.org/10.1111/jfb.12680

Morales, P. \& Dunker, F. (1999). Suspected intestinal torsion in a blacktip reef shark (Carcharhinus melanopterus). Journal of Zoo and Wildlife Medicine, 30(1), 170-172. https://www.jstor.org/stable/20095838

Moss, S. A. (1984). Sharks: An Introduction for the Amateur Naturalist. Prentice-Hall Direct. 246p.

Moura, M. L. A.; Cunha, F. T.; de Macedo, H. J. A.; Batista, J. D. D. O.; Reis, S. M.; Oliveira, S. S. \& Otani, F. S. (2018). Rigor mortis e aspectos reprodutivos de acaris bodós capturados no rio Tapajós, Pará, Brasil. Revista Agroecossistemas, 10(2), 309-315. http://dx.doi.org/10.18542/ragros.v10i2.5170

Mulas, A.; Bellodi, A.; Porcu, C.; Cau, A.; Coluccia, E.; Demurtas, R.; Marongiu, M.F.; Pesci, P. \& Follesa, M.C. (2020). Living naked: first case of lack of skin-related structures in an elasmobranch, the blackmouth catshark (Galeus melastomus). Journal of Fish Biology, 97(4), 1252-1256. https://doi.org/10.1111/jfb. 14468

Noakes, D. E.; Parkinson, T. J.; England, G. C. W. \& Arthur, G. H. (2001). Arthur's veterinary reproduction and obstetrics. Saunders Elsevier. 844p.

Oetterer, M.; Galvão, J. A. \& Savay-Da-Silva, L. K. (2014). Qualidade do Pescado: Sistemas para Padronização. In: Galvão, J. A. \& Oetterer, M. Qualidade e Processamento de Pescado. Rio de Janeiro: Elsevier, p.31-72.

Oddone, M. C. \& Amorim, A. F. (2008). Size at maturity of Atlantoraja platana (Günther, 1880)(Chondrichthyes: Rajidae: Arhynchobatinae) in the south-west Atlantic Ocean. Journal of Fish Biology, 72(6), 1515-1519. http://dx.doi.org/10.1111/j.1095-8649.2007.01774.x

Paragó, C. L. D. P. (2001). Contribuição à taxonomia do gênero Psammobatis Günther, 1870 (Chondrichthyes, Rajidae): Caracterização das espécies do subgrupo I de Mceachran (1983) com base em padrões de coloração e espinulação. (Dissertação do Programa de Pós-graduação em Zoologia da Universidade Federal do Rio de Janeiro.) 106p. https://minerva.ufrj.br/F/?func=direct\&doc_number=000542803\&local_base=UFR01 
Pastén-Marambio, V.; Hevia-Hormazábal, V.; Acuña, E. \& Vega, A. (2018). A case of tetrophthalmia with unilateral synophthalmia in an unborn fetus of blue shark Prionace glauca (Carcharhiniformes, Carcharhinidae). Revista de Biología Marina y Oceanografía, 53, 25-30. https://doi.org/10.22370/rbmo.2018.53.0.1251

Petrini, B. (2006). Mycobacterium marinum: ubiquitous agent of waterborne granulomatous skin infections. European Journal of Clinical Microbiology and Infectious Diseases, 25(10), 609-613. https://doi.org/10.1007/s10096-006-0201-4

Pollom, R.; Barreto, R.; Charvet, P.; Chiaramonte, G.E.; Cuevas, J.M.; Faria, V.; Herman, K.; Montealegre-Quijano, S.; Motta, F.; Paesch, L. \& Rincon, G. (2020). Psammobatis rutrum. The IUCN Red List of Threatened Species 2020. e.T63101A3117368. https://dx.doi.org/10.2305/iucn.uk.2020-3.rlts. t63101a3117368.en

Pracheil, B. M.; DeRolph, C. R.; Schramm, M. P. \& Bevelhimer, M. S. (2016). A fish-eye view of riverine hydropower systems: the current understanding of the biological response to turbine passage. Reviews in fish biology and fisheries, 26(2), 153-167. https://doi.org/10.1007/s11160-015-9416-8

Quigley, D. T. G.; Carlos, A.; Barros-Garcia, D. \& MacGabhann, D. (2018). Albinism and leucism in blonde rays (Raja brachyura Lafont, 1871)(Elasmobranchii: Batoidea) from the Irish Sea. Bulletin of the European Association of Fish Pathologists,38(2), 79-88. https://www.cabdirect.org/cabdirect/abstract/20183186500

Rafrafi-Nouira, S.; El Kamel-Moutalibi, O.; Amor, K. O. B.; Amor, M. M. B. \& Capapé, C. (2017). A case of hermaphroditism in the common eagle ray Myliobatis aquila (Chondrichthyes: Myliobatidae), reported from the Tunisian coast (Central Mediterranean). Series Historia Naturalis, 27(1), 43-48. http://dx.doi.org/10.19233/ASHN.2017.06

Ranjith, L.; Saravanan, R.; Kalidas, C.; Kavitha, M.; Ramkumar, S.; Joshi, K. K. \& Manojkumar, P. P. (2019). Morphological Deformities in Neotrygon kuhlii (Muller \& Henle, 1841) from Gulf of Mannar, Bay of Bengal, India. International Journal of Marine Sciences, 35(1), 49-56. https://doi.org/10.1007/s41208018-0083-0

Regev, Y.; Davidovich, N.; Berzak, R.; Lau, S. C.; Scheinin, A. P.; Tchernov, D. \& Morick, D. (2020). Molecular Identification and characterization of Vibrio species and Mycobacterium species in wild and cultured marine fish from the Eastern Mediterranean Sea. Microorganisms, 8(6), 863. https://doi.org/10.3390/microorganisms 8060863

Ribeiro-Prado, C. C.; Oddone, M. C.; de Amorim, A. F. \& Capape, C. (2009). An abnormal hermaphrodite pelagic stingray Pteroplatytrygon violacea (Dasyatidae) captured off the southern coast of Brazil. Cahiers de Biologie Marine, 50(1), 91-96. https://dx.doi.org/10.21411/CBM.A.A486D4DA

Rocha, M. L. F. \& Dias, J. F. (2015). Inventory of Chondrichthyes and Actinopterygii species collected in the central coast of São Paulo State, Brazil. Biota Neotropica, 15(2), e20140136. https://dx.doi.org/10.1590/1676-06032015013614

Rolim, F. A.; Rotundo, M. M.; Stabile, G. C. \& Vaske-Júnior, T. (2020). Records of morphological abnormalities in the Brazilian electric ray Narcine brasiliensis (Olfers, 1831)(Chondrichthyes: Narcinidae) in the southwestern Atlantic. Marine Biodiversity, 50, 1-7. https://doi.org/10.1007/s12526-020-01072$\mathrm{w}$

Rummer, J. L. (2007). Factors affecting catch and release (CAR) mortality in fish: insight into CAR mortality in red snapper and the influence of catastrophic decompression. American Fisheries Society Symposium, 60, 113-132. https://doi.org/10.47886/9781888569971.ch8

Rummer, J. L. \& Bennett, W. A. (2005). Physiological effects of swim bladder overexpansion and catastrophic decompression on red snapper. Transactions of the American Fisheries Society, 134, 1457-1470. https://doi.org/10.1577/T04-235.1

Sandoval-Castillo, J.; Mariano-Melendez, E. \& Villavicencio-Garayzar, C. (2006). New records of albinism in two elasmobranchs: the tiger shark Galeocerdo cuvier and the giant electric ray Narcine entemedor. Cybium, 30(2), 191-192. https://doi.org/10.26028/cybium/2006-302-014

Santander-Neto, J. \& Lessa, R. (2013). Hermaphroditic smalleyed roundray (Urotrygon microphthalmum) from north-eastern Brazil. Marine Biodiversity Records, 6, 1-4. https://doi.org/10.1017/S1755267213000353

Schaller, P. \& Dunker, F. (2005). Use of contrast radiograph to determine trauma to the valvular intestine in the blacktip reef shark (Carcharhinus melanopterus). Drum and Croaker, 36, 23-28. http://drumandcroaker.org/pdf/2005.pdf

Schimpf, M. \& Tulikangas, P. (2005). Evolution of the female pelvis and relationships to pelvic organ prolapse. International Urogynecology Journal, 16(4), 315-320. https://doi.org/10.1007/s00192-004-1258-1

Schreiber, C. \& Coco, C. (2017). Husbandry of Whale Sharks In: Smith, M.; Warmolts, D.; Thoney, D.; Hueter, R.; Murray, M. \& Ezcurra, J. The Elasmobranch husbandry manual II: recent advances in the care of sharks, rays and their relatives. The Ohio Biological Survey, Columbus. p.87-98.

Schroeder, R.; Pio, V. M.; Bail, G. C., Lopes, F. \& Wahrlich, R. (2016). Análise espaço-temporal da composição da captura da pesca com emalhe de fundo no Sudeste / Sul do Brasil. Boletim do Instituto de Pesca, 40,323-353. https://www.pesca.sp.gov.br/40_3-323-353.pdf

Sen, S.; Dash, G.; Valappil, A. K.; Kizhakudan, S. J. \& Chakraborty, R. (2020). Occurrences of intersexual hound sharks, Iago cf. omanensis (Triakidae: Carcharhiniformes) from north-western Bay of Bengal. Thalassas: An International Journal of Marine Sciences, 36, 525-534. https://doi.org/10.1007/s41208020-00220-0

Silva, J. P. C. B. \& Silva-Casas, A. L. (2020). Morphological deformities in the pelvic fin and clasper in specimens of Potamotrygon marquesi (Chondrichthyes: Myliobatiformes: Potamotrygoninae). Journal of Applied Ichthyology, 36(2), 189-196. https://doi.org/10.1111/jai.14005

Silva-Presenza, L.; dos Santos, A. S.; Bernabé, C. V.; Silva, A. C. F. S.; Ferreira, J. V. F.; de Paiva Garcia, R. C. \& Minozzo, M. G. (2020). Determinação do índice de rigor mortis do juvenil de beijupirá (Rachycentron canadum) utilizando eugenol. Brazilian Journal of Development, 6(1), 5140-5148. http://dx.doi.org/10.34117/bjdv6n1-372

Sims, D. W.; Andrews, P. L. R. \& Young, J. Z. (2000). Fish behaviour: stomach rinsing in rays. Nature, 404(6778), 566. https://doi.org/10.1038/35007149 
Siqueira, A. B. D.; Godoy, H. P. D.; Moraes, A. C. D. \& Prata, L. F. (2018). Avaliação do índice de rigor mortis e da evolução do pH da musculatura de tilápias do Nilo (Oreochromis nilolicus) durante estocagem em gelo sob refrigeração. Higiene alimentar, 32(282/283), 85-90. https://www.higienealimentar.com.br/wp-content/uploads/2019/04/2018-Julho-Agosto-282-283.pdf

Sladky, K. K. \& Clarke, E. O. (2016). Fish surgery: presurgical preparation and common surgical procedures. The Veterinary Clinics of North America. Exotic Animal Practice, 19(1), 55-76. https://doi.org/10.1016/j.cvex.2015.08.008

Smith, S. A. (1997). Mycobacterial infections in pet fish. Seminars in Avian and Exotic Pet Medicine, 6, 40-45. https://doi.org/10.1016/S1055937X(97)80040-0

Soares, K. M. D. P. \& Gonçalves, A. A. (2012). Qualidade e segurança do pescado. Revista do Instituto Adolfo Lutz, 71(1), 1-10. http://periodicos.ses.sp.bvs.br/scielo.php?script=sci_arttext\&pid=S0073-98552012000100001\&lng=pt\&tlng=pt

Staggs, L. A. (2016). The Use of Misoprostol for Cervical Dilation for a Dystocia in an Atlantic Bottlenose Dolphin (Tursiops truncatus). 47th Annual International Association for Aquatic Animal Medicine Meeting \& Conference, May, 21-26, 2016. Virginia Beach, VA, EUA. https://www.vin.com/doc/?id=7312325\&pid=14818

Stewart, J. (2008). Capture depth related mortality of discarded snapper (Pagrus auratus) and implications for management. Fisheries Research, 90, 289-295. https://doi.org/10.1016/j.fishres.2007.11.003

StJohn, J. \& Syers, C.J. (2005). Mortality of the demersal West Australian dhufish, Glaucosoma hebraicum (Richardson, 1845) following catch and release: the influence of capture depth, venting and hook type. Fisheries Research, 76, 106-116. https://doi.org/10.1016/j.fishres.2005.05.014

Stoskopf, M. (1993). Bacterial diseases of freshwater tropical fishes. Fish Medicine. p.559-563. https://www.umces.edu/sites/default/files/fish.pdf

Straube, N.; Lampert, K. P.; Geiger, M. F.; Weiss, J. D. \& Kirchhauser, J. X. (2016). First record of second-generation facultative parthenogenesis in a vertebrate species, the whitespotted bambooshark Chiloscyllium plagiosum. Journal of Fish Biology, 88(2), 668-675. https://doi.org/10.1111/jfb.12862

Tuttle, A. D.; Burrus, O.; Burkart, M. A.; Scott, P. W.; Stoskopf, M. K. \& Harms, C. A. (2008). Three cases of gastric prolapse through the gill slit in sand tiger sharks, Carcharhinus taurus (Rafinesque). Journal of Fish Diseases, 31(4), 311-315. https://doi.org/10.1111/j.1365-2761.2007.00881.x

Van Zeeland, Y. (2016). Reproductive disease in birds. BSAVA Congress Proceedings 2016, p.81-82. http://dx.doi.org/10.22233/9781910443446.6.2

Veldhuizen, L. J. L.; Berentsen, P. B. M.; De Boer, I. J. M.; Van De Vis, J. W. \& Bokkers, E. A. M. (2018). Fish welfare in capture fisheries: A review of injuries and mortality. Fisheries Research, 204, 41-48. https://doi.org/10.1016/j.fishres.2018.02.001

Viana, A. F.; Valentin, J. L. \& Vianna, M. (2017). Feeding ecology of elasmobranch species in southeastern Brazil. Neotropical Ichthyology, $15(2)$, e160176. https://dx.doi.org/10.1590/1982-0224-20160176

Yanong, R. P., Curtis, E. W., Terrell, S. P., \& Case, G. (2003). Atypical presentation of mycobacteriosis in a collection of frogfish (Antennarius striatus). Journal of Zoo and Wildlife Medicine: official publication of the American Association of Zoo Veterinarians, 34(4), 400-407. https://doi.org/10.1638/02-050

Young, J. Z. (1983). Control of movements of the stomach and spiral intestine of Raja and Scyliorhinus. Journal od the Marine Biological Association oh the United Kingdom, 63(3), 557-574. https://doi.org/10.1017/S0025315400070879

Zanini, M.S.; Martins, J.D.; Torres, A. \& Tobias, F.L. (2001). Avaliação microbiológica do gelo de balcão frigorífico de peixarias da grande VitóriaES. Higiene Alimentar, 15(80/81), 122.

Zhao, Y.; Chen, M.; Jiang, C.; Yang, S. \& Xiao, J. (2017). Occurrence of an intersexual pacific spadenose shark Scoliodon macrorhynchos from the Southern Taiwan Strait. Marine and Coastal Fisheries, 9(1), 573-576. https://doi.org/10.1080/19425120.2017.1383326

Weideli, O. C. \& Papastamatiou, Y. P. (2021). Intestinal eversion in a pelagic shark evokes chase and bite attempts by large-bodied carangid fish. Journal of Ethology, 39(1), 125-127. https://doi.org/10.1007/s10164-020-00664-0

Wiersma, G.; Verhoog, P.; Fowler, S. \& Meekan, M. (2016). The first field observation of intestinal eversion by a shark (broadnose sevengill, Notorynchus cepedianus). Marine Biodiversity, 46, 17-18. https://doi.org/10.1007/s12526-015-0341-9

Wildgoose, W. H. (2000). Fish surgery: an overview. Fish Veterinary Journal, 5, 22-36. https://www.researchgate.net/publication/310842827_Fish_surgery_ An_overview

Wosnick, N.; Adams, K. R. \& Freire, C. A. (2018). Ultrasonography as a promising methodology to indicate captured-induced abortion in viviparous elasmobranchs. Journal of Fish Biology, 93(6), 1033-1037. https://doi.org/10.1111/jfb.13746

Wosnick, N.; Awruch, C. A.; Adams, K. R.; Gutierre, S. M. M.; Bornatowski, H.; Prado, A. C. \& Freire, C. A. (2019). Impacts of fisheries on elasmobranch reproduction: high rates of abortion and subsequent maternal mortality in the shortnose guitarfish. Animal Conservation, 22(2), 198-206. https://doi.org/10.1111/acv.12458 